\title{
Прогнозирование жизненного цикла инновации с учетом влияния инфляции
}

\author{
Клещева O.A. ${ }^{1}$ \\ ${ }^{1}$ Казанский государственный архитектурно-строительный университет, Казань, Россия
}

\begin{abstract}
АННОТАЦИЯ:
В данный момент проблема обоснования инновационных проектов приобретает особую актуальность. Предприятиям и организациям необходимо знать величину отдачи на вложенный капитал на десятилетия вперед. Особенный интерес представляет прогнозирование денежных потоков от реализации инновационных проектов, так как в России существует большой потенциал для роста инновационной активности организаций. В процессе прогнозирования жизненного цикла инновации на долгосрочный период основными вопросами являются прогнозирование спроса и инфляции. В статье решается задача прогнозирования инфляции при расчете жизненного цикла инновации. В результате анализа была предложена регрессионная модель прогнозирования инфляции. Выводы, изложенные в статье, могут быть использованы на практике в процессе прогнозирования жизненного цикла инновации.
\end{abstract}

КЛЮЧЕВЫЕ СЛовА: инновации, жизненный цикл инновационного проекта, прогнозирование инфляции.

Forecasting the life cycle of innovation, taking into account the impact of inflation

Klescheva O.A. ${ }^{1}$

${ }^{1}$ Kazan State University of Architecture and Engineering, Russia

\section{Введение}

$\mathrm{B}$ настоящее время возрастает роль планирования и прогнозирования экономического развития как на макроуровне, так и на микроуровне. Предприятиям и организациям необходимо знать величину отдачи на вложенный капитал на десятилетия вперед [1] (Bakhareva, Kordonchik, 2016). Особенный интерес представляет прогнозирование денежных потоков от реализации инновационных проектов [4] (Zagidullina, Nizamova, 2015). Наибольший интерес для предприятий представляют инновационные проекты, которые при относительно небольших инвестициях смогут создать конкурентоспособную продукцию [10] (Sayfullina, 2010). В России существует большой потенциал для роста инновационной активности организаций [9] (Romanova, 2018) (puc. 1).

В современных условиях особенно важно обеспечить внедрение инноваций не только на крупных, но и на малых предприятиях [3] 
(Dobroserdova, Nizamova, 2015). Именно необходимость крупных вложений при неопределенности отдачи на вложенный капитал замедляет процесс внедрения инноваций в некоторых отраслях [13] (Fayzullina, Azhimov, 2011). Разработка методики прогнозирования жизненного цикла инновационного проекта позволит снизить неопределенность инновационной деятельности, а следовательно, повысит ее привлекательность.

\section{Методика}

В процессе прогнозирования жизненного цикла инновации на долгосрочный период основными вопросами являются прогнозирование спроса и инфляции. В процессе прогнозирования спроса на инновацию необходимо опираться на данные маркетинговых исследований, учитывать известные в экономической литературе факторы, влияющие на изменение спроса.

Прогнозированию инфляции посвящена работа [16] (Shumetov, Afonina, 2013). Авторы анализируют индекс потребительских цен - показатель, применяемый для оценки уровня инфляции. В прогнозной модели факторами, определяющими значение индекса потребительских цен, являются индексы цен на продовольственные и непродовольственные товары и на услуги. В работе [11] (Sedelnikov, Kondratieva, 2004) уровень инфляции прогнозируется с помощью метода замены переменных. А. И. Кузовкин построил модель, учитывающую влияние на инфляцию цен на газ [6]. В статье Э. С. Новиковой и С. С. Федорова подробно анализируются причины инфляции,

\section{ABSTRACT:}

At the moment, the problem of justifying innovative projects is becoming especially urgent. Enterprises and organizations need to know the amount of return on invested capital for decades to come. Of particular interest is the forecasting of cash flows from the implementation of innovative projects, as in Russia there is a great potential for growth of innovative activity of organizations. In the process of forecasting the life cycle of innovation for a long-term period, the main issues are forecasting demand and inflation. The article solves the problem of forecasting inflation in the calculation of the life cycle of innovation. As a result of the analysis, a regression model of inflation forecasting was proposed. The conclusions stated in the article can be used in practice in the process of forecasting the life cycle of innovation.

KEYWORDS: innovation, life cycle of the innovation project, forecasting of inflation.

JEL Classification: 031, 032, 033

Received: 27.08.2018 / Published: 30.09.2018

(c) Author(s) / Publication: CREATIVE ECONOMY Publishers

For correspondence: Klescheva O.A. (olga_albertovnalabk.ru)

\section{CITATION:}

Klescheva O.A. (2018) Prognozirovanie zhiznennogo tsikla innovatsii s uchetom vliyaniya inflyatsii [Forecasting the life cycle of innovation, taking into account the impact of inflation]. Voprosy innovatsionnoy ekonomiki. 8. (3). - 405-416. doi: 10.18334/vinec.8.3.39351 


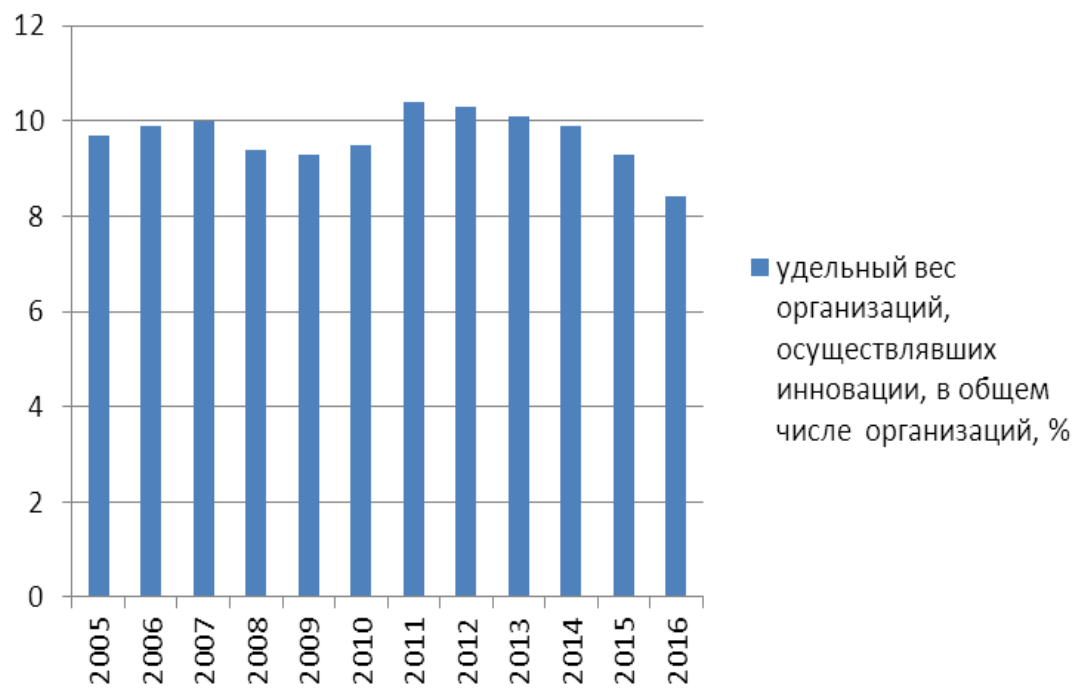

Рисунок 1. Совокупный уровень инновационной активности организаций в России Источник: данные Федеральной службы государственной статистики [14]

делается вывод, что основные причины инфляции - это высокая степень монополизации рынка и слабая связь заработной платы и производительности труда [7] (Novikova, Fedorov, 2013). О. А. Патваканян обосновывает применение марковских цепей для прогноза инфляции [8] (Patvakanyan, 2016). Он пишет о необходимости применения нелинейных моделей для прогнозирования инфляции.

При прогнозировании и моделировании экономических процессов авторы используют различные методы. Например, в работе [12] (Suvorov, 2015) рассматривается линейная регрессионная модель с переменными во времени структурными параметрами; в исследовании [5] (Kossov, 2016) для моделирования применяется экспоненциальная и логарифмическая зависимость; в работе [2] (Bogdanov, Ilysheva, Baldesku, Zakirov, 2016) так же используется логарифмическая зависимость.

В процессе прогнозирования инфляции используют методы экстраполяции. Используя степенную и логарифмическую зависимость, на основе данных по индексам потребительских цен по России был сделан прогноз инфляции (рuс. 2).

\section{ОБ АВTOPE:}

Клещева Ольга Альбертовна, кандидат экономических наук, доцент кафедры экономики и предпринимательства в строительстве (olga_albertovnalabk.ru)

\section{ЦИТИРОВАТЬ СТАТЬЮ:}

Клещева О.А. Прогнозирование жизненного цикла инновации с учетом влияния инфляции // Вопросы инновационной экономики. - 2018. - Том 8. - № 3. - С. 405-416. doi: 10.18334/vinec.8.3.39351 


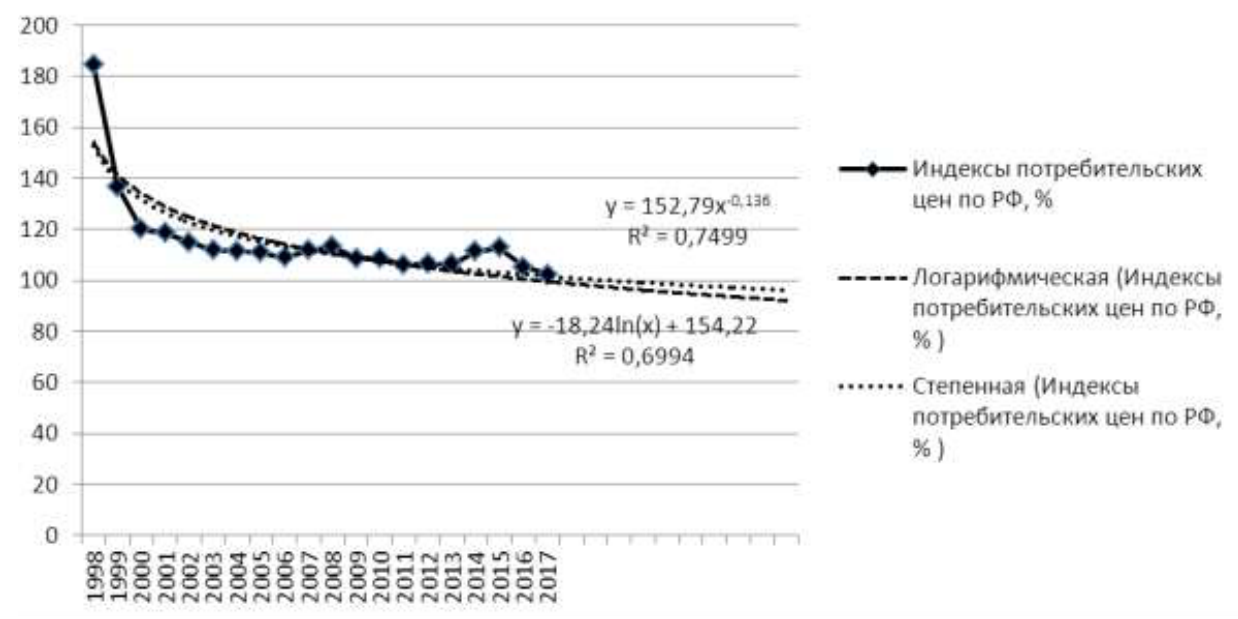

Рисунок. 2. Прогноз инфляции на основе экстраполяции показателя «Индекс потребительских цен по РФ»

Источник: составлено автором на основе [14]

Используя одномерные зависимости трудно добиться высокой точности прогноза. Позтому для прогнозирования инфляции мы предлагаем использовать метод регрессии (проекции) на латентные структуры (РЛС) [17] (Esbensen, 2008). В процессе расчетов модели РЛС матрица зависимой переменной и матрица факторов, оказывающих влияние на зависимую переменную, подвергаются одновременному анализу с помощью метода главных компонент, а затем строится уравнение регрессии.

\section{Результаты и обсуждения}

В процессе анализа были использованы данные по параметрам, оказывающим влияние на инфляционные процессы в России (табл. 1).

В процессе построения модели, в первую очередь, определяется число используемых главных компонент с помощью анализа остаточной дисперсии. Две первые главные компоненты объясняют 75\% вариации показателя «Индексы потребительских цен по РФ». В процессе построения уравнения методом РЛС рассчитаны значения векторов нагрузок [17] (табл. 2).

Значение вектора нагрузки фактора определяет его влияние на вектор главной компоненты.

На направление вектора первой главной компоненты наибольшее влияние имеют (табл. 2, рис. 3): индексы потребительских цен; валовой внутренний продукт; ключевая ставка ЦБ; курс доллара; инвестиции в основной капитал. Направление вектора второй главной компоненты определяется следующими факторами: индексы потре- 


\begin{tabular}{|c|c|c|c|c|c|c|c|}
\hline \multirow{21}{*}{ 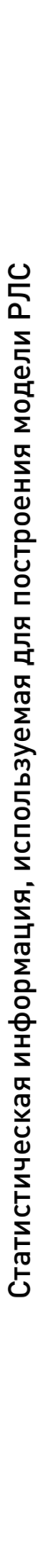 } & $\angle 102$ & lg'Z0l & 乙'LEOZ6 & †レ'6 & $\varepsilon^{\prime} 89$ & El'86 & $0 \varepsilon^{\prime} 980 \angle 07$ \\
\hline & 9102 & $6 \varepsilon^{\prime} 901$ & $9 ' 87198$ & 9'0l & $\varepsilon 0^{\prime} \angle 9$ & l'lol & 06'9サ88クレサレ \\
\hline & sloz & $16 ' 211$ & $Z^{\prime} \angle 8 \varepsilon \varepsilon 8$ & $9 Z^{\prime} 8$ & 96109 & 9'96 & OL'L8LL68EL \\
\hline & 7102 & ૬E'เル & $L ' 6616 L$ & G'0l & $19^{\prime} 8 \varepsilon$ & L'lOL & 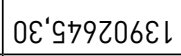 \\
\hline & Eloz & Lサ'90L & 6'EElEL & $G^{\prime} g$ & $98^{\prime} l \varepsilon$ & †'00l & 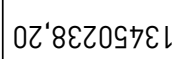 \\
\hline & Zloz & $\angle G^{\prime} 90 \mathrm{~L}$ & 6'E9l89 & $9 \nearrow^{\prime} 8$ & $80^{\prime} l \varepsilon$ & $\eta^{\prime} \varepsilon 0 l$ & 0サ'060989Z। \\
\hline & ILOZ & l'90l & G'Z8Z09 & 8 & $6 \varepsilon^{\prime} 62$ & sol & 00'Z99980l \\
\hline & 0102 & $8 L^{\prime} 80 L$ & G'80ع9† & $G L^{\prime} L$ & $9 \varepsilon^{\prime} 0 \varepsilon$ & $\varepsilon^{\prime} \angle O L$ & $00 ' 9602916$ \\
\hline & 6002 & $8^{\prime} 80 l$ & Z'L088E & Zl & $\varsigma^{\prime} 0 \varepsilon$ & $\varepsilon^{\prime} 68$ & 08'ZlO9L6L \\
\hline & 8002 & 8Z'દlı & 8'9டてい & ll & 乙o's乙 & 9'00l & 0サ'91918L8 \\
\hline & $\angle 00 Z$ & L8'ルレ & $G^{\prime} \angle \pitchfork 乙 \varepsilon \varepsilon$ & $\mathrm{Ol}$ & ভ9'ウ乙 & 8'90l & 0サ'てZZ9レ८9 \\
\hline & 9002 & 601 & Z'L169Z & 乙८ & $\varepsilon \mathcal{E}^{\prime} 9 乙$ & $\varepsilon^{\prime} 90 l$ & 06'ZZ૦૦६L† \\
\hline & S00Z & Z6'0ll & $8^{\prime} 60912$ & $\varepsilon l$ & $8 L^{\prime} 8 Z$ & l'sol & 00'60lll9e \\
\hline & 7002 & EL'ルl & Z'LZOLL & サレ & $G L ' L Z$ & $80 l$ & 06 'ElOS98Z \\
\hline & $\varepsilon 00 Z$ & 66'lll & 乙'80乙El & $\angle L$ & $97{ }^{\prime} 62$ & 6'80l & oZ'ง9ع98l乙 \\
\hline & Z00乙 & 90'งl & $\mathrm{s}^{\prime} 0 \varepsilon 80 \mathrm{l}$ & $\varepsilon 乙$ & $8 L^{\prime} l \varepsilon$ & l'EOl & OE'LOサZ9LL \\
\hline & 1002 & $89^{\prime} 8 \mathrm{ll}$ & $9^{\prime} \varepsilon \ni 68$ & 92 & †レ'0ع & 6'ZOL & 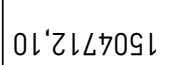 \\
\hline & 0002 & 8 8’0Zl & 9'GOEL & $\varepsilon \varepsilon$ & $91 ' 8 Z$ & $L ' 80 L$ & 0Z'ゥยて૬9レ \\
\hline & 6661 & $\varepsilon G^{\prime} 9 \varepsilon l$ & 乙'EZ৪૪ & $\angle G$ & $69^{\prime}+2$ & 6'80I & 08'8ع†0८9 \\
\hline & $866 l$ & ยザサ8l & 96292 & $\varepsilon G$ & s9'0Z & Z'96 & $0 \varepsilon^{\prime} 980 \angle 07$ \\
\hline & 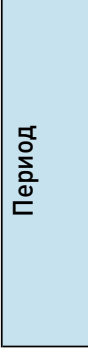 & 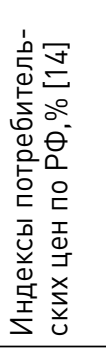 & 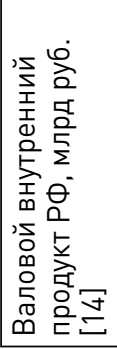 & 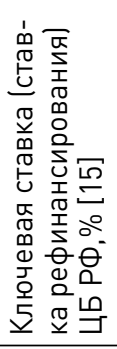 & 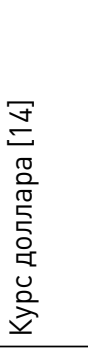 & 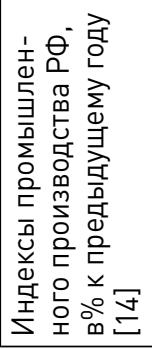 & 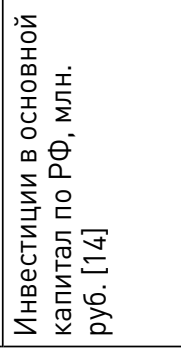 \\
\hline
\end{tabular}


Величина векторов нагрузок для модели РлС

Таблица 2

\begin{tabular}{|l|c|c|}
\hline \multicolumn{1}{|c|}{ Показатели } & ГК1 & ГК2 \\
\hline Индексы потребительских цен по РФ,\% & 0,479 & 0,447 \\
\hline Валовой внутренний продукт РФ, млрд руб. & $-0,587$ & 0,297 \\
\hline Ключевая ставка (ставка рефинансирования) ЦБ РФ,\% & 0,57 & 0,281 \\
\hline Курс доллара & $-0,431$ & 0,38 \\
\hline Индексы промышленного производства РФ, в\% К предыдущему году & 0,127 & $-0,857$ \\
\hline Инвестиции в основной капитал по РФ, млн руб. & $-0,532$ & 0,199 \\
\hline
\end{tabular}

Источник: составлено автором

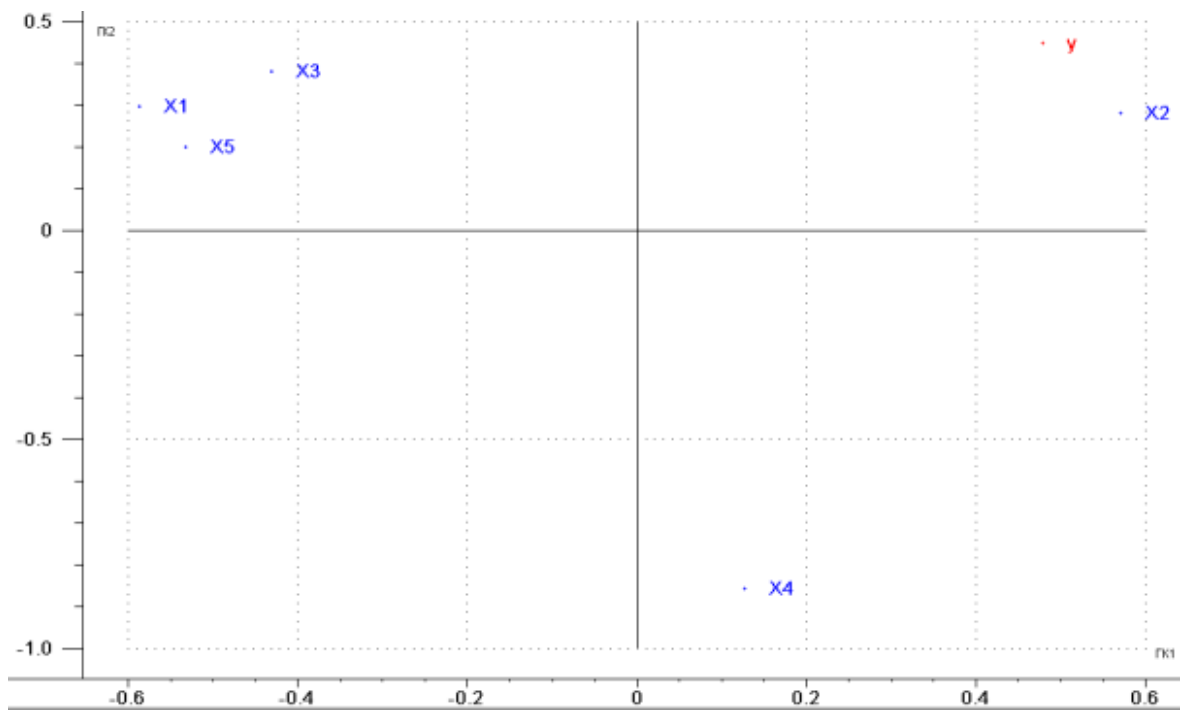

Рисунок 3. Значения нагрузок для модели РЛС

Источник: составлено автором

Обозначения: у - индексы потребительских цен по РФ,\%; $\mathrm{x}_{1}$ - валовой внутренний продукт РФ,

млрд руб.; $\mathrm{x}_{2}$ - ключевая ставка (ставка рефинансирования) ЦБ РФ,\%; $\mathrm{x}_{3}$ - курс доллара; $\mathrm{x}_{4}$ индексы промышленного производства РФ, в\% к предыдущему году; $\mathrm{x}_{5}$ - инвестиции в основной капитал по РФ, млн руб.

бительских цен; индексы промышленного производства. Показатель «индекс потребительских цен по РФ» имеет высокий положительный коэффициент корреляции с показателем «ключевая ставка (ставка рефинансирования) ЦБ РФ» и отрицательный коэффициент корреляции с показателями «валовой внутренний продукт РФ», «курс доллара», «инвестиции в основной капитал по РФ». 
По итогам анализа рассчитана следующая регрессионная модель:

$$
y=0,00008^{\star} x_{1}+0,799^{\star} x_{2}+0,113^{\star} x_{3}-1,401^{*} x_{4}-0,0000003^{\star} x_{5}+254,67,
$$

Сопоставление значений, рассчитанных с помощью модели, с реальной величиной показателя «Индекс потребительских цен по РФ» представлены в таблице 3 и на рисунке 4 .

Таблица 3

Значения показателя «Индекс потребительских цен РФ» для модели РЛС

\begin{tabular}{|c|c|c|c|}
\hline Период & $\begin{array}{c}\text { Индексы потребитель- } \\
\text { ских цен по РФ,\% }\end{array}$ & $\begin{array}{l}\text { Индекс потребительских } \\
\text { цен по РФ, определенный } \\
\text { на основе модели РЛС,\% }\end{array}$ & Расхождение значений,\% \\
\hline 1998 & 184,43 & 160,99 & $-13 \%$ \\
\hline 1999 & 136,53 & 144,31 & $6 \%$ \\
\hline 2000 & 120,18 & 124,69 & $4 \%$ \\
\hline 2001 & 118,58 & 125,98 & $6 \%$ \\
\hline 2002 & 115,06 & 123,70 & $8 \%$ \\
\hline 2003 & 111,99 & 110,75 & $-1 \%$ \\
\hline 2004 & 111,73 & 109,32 & $-2 \%$ \\
\hline 2005 & 110,92 & 111,92 & $1 \%$ \\
\hline 2006 & 109 & 109,01 & $0 \%$ \\
\hline 2007 & 111,87 & 105,90 & $-5 \%$ \\
\hline 2008 & 113,28 & 114,17 & $1 \%$ \\
\hline 2009 & 108,8 & 130,58 & $20 \%$ \\
\hline 2010 & 108,78 & 101,08 & $-7 \%$ \\
\hline 2011 & 106,1 & 103,02 & $-3 \%$ \\
\hline 2012 & 106,57 & 104,25 & $-2 \%$ \\
\hline 2013 & 106,47 & 105,55 & $-1 \%$ \\
\hline 2014 & 111,35 & 106,36 & $-4 \%$ \\
\hline 2015 & 112,91 & 108,85 & $-4 \%$ \\
\hline 2016 & 105,39 & 103,30 & $-2 \%$ \\
\hline 2017 & 102,51 & 110,44 & $8 \%$ \\
\hline
\end{tabular}

Источник: составлено автором

Разность в величине значений показателя «Индекс потребительских цен по РФ» вызвана воздействием параметров, которые не были учтены при моделировании. 


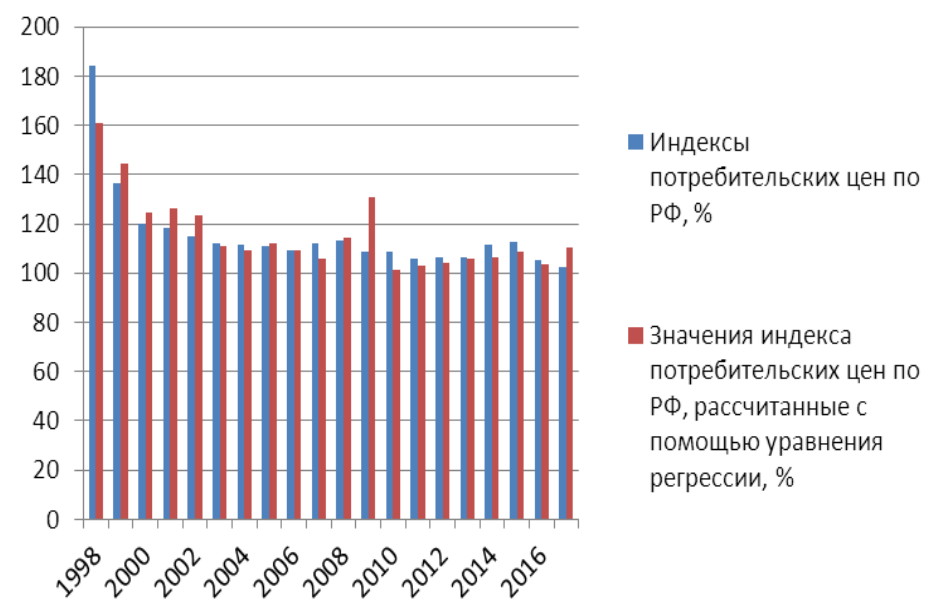

Рисунок 4. Значения показателя «Индекс потребительских цен по РФ» для модели РЛС Источник: составлено автором

\section{Заключение}

Таким образом, по результатам проведенного анализа можно сделать вывод о возможности прогнозирования жизненного цикла инновационного продукта с использованием моделирования величины инфляции. Как показывают проведенные исследования, величина инфляции подвержена воздействию множества экономических факторов. Основное влияние оказывают следующие факторы: валовой внутренний продукт; ключевая ставка ЦБ; курс доллара и инвестиции в основной капитал.

\section{ИСТОЧНИКИ:}

1. Бахарева О.В., Кордончик Д.М. Институты развития в инновационной структуре региона // Управление экономическими системами: электронный научный журнал, 2016. - № 12(94).

2. Богданов В.Д., Илышева Н.Н., Балдеску Е.В., Закиров У.Ш. Модель корреляции между экономическим развитием и экологической результативностью на основе данных нефинансовой отчетности компании // Экономика региона, 2016. - № 1. - doi: 10.17059/2016-1-7.

3. Добросердова Е.А., Низамова А.Ш. Реализация концепции инвестиционно-инновационного лифта для предприятий малого бизнеса в условиях современной экономической ситуации // Вестник экономики, права и социологии. 2015. - № 2.

4. Загидуллина Г.М., Низамова И.Р. Механизмы государственного стимулирования коммерциализации инноваций // Управление экономическими системами: электронный научный журнал, 2015. - № 10 (82). 
5. Коссов В.В. Обоснование прогнозируемой цены спроса на электроэнергию для промышленности России до 2020 г // Проблемы прогнозирования, 2016. - № 1(154).

6. Кузовкин А.И. Прогноз и оценка влияния цен на газ на уровень инфляции и ВВП Российской Федерации // Микроэкономика, 2009. - № 4.

7. Новикова Э.С., Федоров С.С. Факторы и прогноз темпов инфляции в России // Современные проблемы экономического и социального развития, 2013. - № 9.

8. Патваканян О.А. Применение марковских цепей для прогноза инфляции в Армении // Вестник Российско-Армянского (Славянского) университета: гуманитарные и общественные науки, 2016. - № 1.

9. Романова А.И. Роль инновационных территориальных кластеров в реализации инновационной политики Республики Татарстан // Сибирская финансовая школа, 2018. - № 2 (127).

10. Сайфуллина Ф.М. К вершинам инновационности в строительстве. Экономические и управленческие аспекты повышения инновационной активности строительных предприятий // Креативная экономика, 2010. - № 10(46).

11. Седельников А.В., Кондратьева И.А. Моделирование уровня ежегодной инфляции в России методом замены переменных // Успехи современного естествознания, 2004. - № 7.

12. Суворов Н.В. Актуальные направления и проблемы совершенствования модельного инструментария макроэкономического анализа // Проблемы прогнозирования, 2015. - № 5 (152).

13. Файзуллина Л.И., Ажимов Т.3. Перспективные внедрения инноваций в строительном кластере// Управление экономическими системами: электронный научный журнал, 2011. - № 12(36).

14. Федеральная служба государственной статистики. [Электронный ресурс]. URL: http://www.gks.ru (дата обращения: 02.08.2018).

15. Центральный банк Российской Федерации [Электронный ресурс]. URL: http://www. cbr.ru (дата обращения: 12.08.2018).

16. Шуметов В.Г., Афонина Т.Н. Инфляция в зеркале статистики: ретроспективный анализ и возможности прогноза // Среднерусский вестник общественных наук, 2013. - № 3(29).

17. Эсбенсен К. Анализ многомерных данных / Избранные главы. - Казань: изд-во КазГАСУ, 2008.

\section{REFERENCES:}

Bakhareva O.V., Kordonchik D.M. (2016). Instituty razvitiya v innovatsionnoy strukture regiona [Development institutions in the innovation structure of the region]. Management of economic systems: scientific electronic journal. (12(94)). (in Russian). 
Bogdanov V.D., Ilysheva N.N., Baldesku E.V., Zakirov U.Sh. (2016). Model korrelyatsii mezhdu ekonomicheskim razvitiem i ekologicheskoy rezultativnostyu na osnove dannyh nefinansovoy otchetnosti kompanii [The Development of Correlation Model between Economic Development and Environmental Performance on the Basis of Non-Financial Reporting]. Economy of the region. 12 (1). (in Russian). doi: 10.17059/2016-1-7.

Dobroserdova E.A., Nizamova A.Sh. (2015). Realizatsiya kontseptsii investitsionnoinnovatsionnogo lifta dlya predpriyatiy malogo biznesa $\mathrm{v}$ usloviyakh sovremennoy ekonomicheskoy situatsii [Implementation of Conception of Investment and Innovations Lift for Small Business in Modern Economic Situation]. The Review of Economy, the Law and Sociology. (2). (in Russian).

Esbensen K. (2008). Analiz mnogomernyh dannyh [Multidimensional data analysis] Kazan: izd-vo KazGASU. (in Russian).

Fayzullina L.I., Azhimov T.Z. (2011). Perspektivnye vnedreniya innovatsiy v stroitelnom klastere [Promising innovations in the construction cluster]. Management of economic systems: scientific electronic journal. (12(36)). (in Russian).

Kossov V.V. (2016). Obosnovanie prognoziruemoy tseny sprosa na elektroenergiyu dlya promyshlennosti Rossii do $2020 \mathrm{~g}$ [Justification of the projected electricity demand price for the Russian industry until 2020]. Problems of forecasting. (1(154)). (in Russian).

Kuzovkin A.I. (2009). Prognoz i otsenka vliyaniya tsen na gaz na uroven inflyatsii i VVP Rossiyskoy Federatsii [Forecast and assessment of the impact of gas prices on inflation and GDP of the Russian Federation]. Microeconomics. (4). (in Russian).

Novikova E.S., Fedorov S.S. (2013). Faktory i prognoz tempov inflyatsii v Rossii [Factors and forecast of inflation rates in Russia]. Sovremennye problemy ekonomicheskogo i sotsialnogo razvitiya. (9). (in Russian).

Patvakanyan O.A. (2016). Primenenie markovskikh tsepey dlya prognoza inflyatsii v Armenii [Application of markov chains to forecast inflation in armenia]. Vestnik Rossiysko-Armyanskogo (Slavyanskogo) universiteta: gumanitarnye i obschestvennye nauki. (1). (in Russian).

Romanova A.I. (2018). Rol innovatsionnyh territorialnyh klasterov v realizatsii innovatsionnoy politiki Respubliki Tatarstan [Role of innovative territorial clusters in realization of innovative policy of the republic of tatarstan]. Siberian Financial School. (2(127)). (in Russian).

Sayfullina F.M. (2010). K vershinam innovatsionnosti v stroitelstve. Ekonomicheskie i upravlencheskie aspekty povysheniya innovatsionnoy aktivnosti stroitelnyh predpriyatiy [Economic and Management aspects of efficiency rise of innovative activity at building enterprises]. Creative economy. (10(46)). (in Russian). 
Sedelnikov A.V., Kondrateva I.A. (2004). Modelirovanie urovnya ezhegodnoy inflyatsii v Rossii metodom zameny peremennyh [Modeling the level of annual inflation in Russia by changing variables]. Advances in current natural sciences. (7). (in Russian).

Shumetov V.G., Afonina T.N. (2013). Inflyatsiya v zerkale statistiki: retrospektivnyy analiz i vozmozhnosti prognoza [Inflation in the mirror of statistics: a retrospective analysis and prognosis possibility]. Central Russian Journal of Social Sciences. (3(29)). (in Russian).

Suvorov N.V. (2015). Aktualnye napravleniya i problemy sovershenstvovaniya modelnogo instrumentariya makroekonomicheskogo analiza [Current trends and problems of improving model tools of macroeconomic analysis]. Problems of forecasting. (5(152)). (in Russian).

Zagidullina G.M., Nizamova I.R. (2015). Mekhanizmy gosudarstvennogo stimulirovaniya kommertsializatsii innovatsiy [Mechanisms of state stimulation of commercialization of innovations]. Management of economic systems: scientific electronic journal. (10(82))/ (in Russian). 
